Itinéraires Itinéraires

Littérature, textes, cultures

2015-1 | 2015

Récits de société

\title{
Autopsies en séries : la transparence et l'ombre
}

\section{Jacqueline Guittard}

\section{OpenEdition}

\section{Journals}

Édition électronique

URL : http://journals.openedition.org/itineraires/2781

DOI : 10.4000/itineraires. 2781

ISSN : 2427-920X

Éditeur

Pléiade

\section{Référence électronique}

Jacqueline Guittard, « Autopsies en séries : la transparence et l'ombre », Itinéraires [En ligne], 2015-1 | 2015, mis en ligne le 18 décembre 2015, consulté le 01 mai 2019. URL : http:// journals.openedition.org/itineraires/2781 ; DOI : 10.4000/itineraires.2781

Ce document a été généré automatiquement le 1 mai 2019.

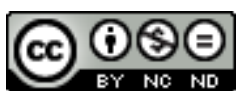

Itinéraires est mis à disposition selon les termes de la licence Creative Commons Attribution - Pas d'Utilisation Commerciale - Pas de Modification 4.0 International. 


\title{
Autopsies en séries : la transparence et l'ombre
}

\author{
Jacqueline Guittard
}

1 Il fut un temps, pas si lointain, où le passage de la vie à la mort s'accomplissait au domicile du défunt, entouré des siens. Fortement ritualisé, il ménageait une prise de congé progressive: exposition du corps sur le lit de mort, masque mortuaire, photographie post-mortem, veillées funèbres, mise en bière, cortège, cérémonie religieuse, enterrement, tout ceci organisé dans un raffinement protocolaire complexe. Ces pratiques ont connu un certain nombre de modifications parmi lesquelles la plus significative fut de soustraire le corps à l'espace familial pour le confier à la fonctionnalité de l'hôpital, de sa morgue et du funérarium. Sans revenir sur les multiples causes qui ont conduit à pareil changement, il convient d'en souligner un effet majeur, largement discuté aujourd'hui dans le champ de la sociologie: la mort serait occultée dans notre société, alors même que cette occultation ne serait pas anthropologiquement désirée.

2 Telle est l'hypothèse de Fabienne Soldini (2010) qui voit «l'engouement pour l'art macabre contemporain » comme une réponse au « désarroi face à la mort charnelle » :

[...] l'art propose un discours mortuaire dans une société où les repères cognitifs traditionnels sur la mort, telles la religion ou l'éthique, semblent défaillants et conduisent les individus à se bricoler des systèmes explicatifs faisant sens.

C'est la raison pour laquelle la mort ferait retour ailleurs, et plus particulièrement dans les séries télévisées policières fondées sur l'autopsie ; immédiatement disponibles au plan de leur réception, elles rapatrient au sein du foyer le cadavre escamoté. Pour avoir effectué une analyse des programmes de la semaine du 22 au 28 janvier 2007, Pierre Mercklé et Thomas Dollé (2009: 229-246) ont pu annoncer que «les téléspectateurs de chaînes françaises gratuites ont eu chaque semaine la possibilité de regarder entre dix et quinze épisodes de séries "macabres" ». En France, Dexter fut l'une des séries les plus remarquées, ayant bénéficié d'une large campagne de communication et d'une audience qui ne l'était pas moins. Son succès a entrainé par contagion la réussite de séries de 
moindre envergure, telles que Bones et Body of proof ${ }^{1}$. Ce sont ces trois œuvres, facilement accessibles au public français, qui serviront de fil rouge à l'examen de la représentation contemporaine du cadavre entre invisibilité et transparence.

\section{Revue de séries}

La série Dexter emprunte son titre au personnage principal, Dexter Morgan ${ }^{2}$. Psychopathe avéré, Dexter est expert scientifique auprès de la police de Miami, spécialisé dans les taches de sang et la façon dont elles maculent une scène de crime. En mode diurne, il travaille, entretient de bonnes relations avec ses collègues, sa sœur Debra, également dans la police, et sa petite amie dont il partage la vie de famille. Mais en mode nocturne, Dexter assouvit des pulsions meurtrières actives depuis l'enfance : à l'âge adulte, il est parvenu à les canaliser et les dirige contre des criminels que le système judiciaire a laissés en liberté, soit parce que l'avocat défendeur a pu faire jouer un vice de procédure, soit parce que la police n'a pas réussi à apporter la preuve de la culpabilité. Il tue ses victimes la nuit dans un espace clandestin - pas toujours le même - aménagé selon les règles en vigueur dans une salle d'autopsie: hygiène, sécurité, praticité. Feuilletonnante, l'architecture de la série Dexter est d'une grande sophistication : elle est construite selon plusieurs niveaux de narration ${ }^{3}$. Le premier niveau s'appuie sur une enquête ponctuelle tandis que le second repose sur les expéditions meurtrières de Dexter : l'une et l'autre seront achevées à la fin de l'épisode. Mais les troisième et quatrième niveaux enjambent toute la saison, formant deux arcs narratifs qui entremêlent la recherche du tueur en série et celle du traumatisme originel de Dexter.

En regard, les deux autres séries sont de facture moins élaborée mais leur simplicité garantit en quelque sorte la visibilité des stéréotypes qu'elles empruntent aux œuvres voisines. Sans les présenter comme des avatars de Dexter - les dates de création ne permettent pas de conclure en ce sens - elles font un peu figure de copies opportunistes sur le marché extrêmement vigoureux et concurrentiel des séries macabres. Bones met en scène une scientifique, anthropologue judiciaire, Temperance Brennan, dont la spécialité consiste à tirer du squelette humain les ultimes indices probants; elle intervient après que toute chair a disparu du cadavre. Elle travaille la plupart du temps en équipe dans un laboratoire ultramoderne, mais elle n'hésite pas à se rendre sur le terrain pour enquêter et parfois se battre telle une héroïne de manga, en compagnie de l'inspecteur Booth qui lui est attitré dans la série ${ }^{4}$. Body of proof, la dernière-née des trois, incarne une chirurgienne devenue incapable d'opérer les vivants. Au sein de son laboratoire, elle est une généraliste de l'autopsie; sur le terrain elle fait équipe avec l'inspecteur Peter Dunlop. Des trois experts légistes, c'est elle qui semble la plus normale : elle est divorcée, mère d'une adolescente, et s'applique à faire face aux difficultés de la vie ordinaire ${ }^{5}$.

Dans la typologie des séries américaines proposées par l'historienne Marjolaine Boutet (2010), la déferlante des séries macabres s'inscrit dans la période postérieure à 1990, qu'elle intitule "réalisme» et "politically incorrectness». Si jusqu'alors, les séries servaient largement l'idéologie dominante, elles s'en écartent en 1990 quand surviennent dans le paysage télévisuel américain les chaînes câblées. Parce qu'elles sont payantes, qu'elles ne sont pas hertziennes, les chaînes câblées n'ont pas à se soumettre aux directives de la FCC (Federal Communications Commission) : c'est là un fait d'importance qui conditionne la production télévisuelle américaine et son effet sur le phénomène de la série macabre. Il convient de rappeler que la FCC, créée en 1934 par le Congrès américain, 
avait pour mission originelle de réguler les télécommunications. Puis sa mission de régulation s'est doublée en 1960 d'une mission de censure toujours en vigueur : les médias ne doivent contenir aucun propos indécent, obscène ou blasphématoire et l'on ne doit pas y montrer la nudité. Le progrès aidant, ses prérogatives se sont étendues à d'autres canaux (radio, télévision, et enfin, Internet) et les interdits s'en sont trouvés d'autant plus diffusés. La première des chaînes à profiter de la brèche ouverte par le câble et à transgresser - légalement - les interdits de la FCC fut la chaîne HBO à laquelle on doit, par exemple, Sex and The City, Six Feet Under, Les Soprano, The Wire, et plus récemment Game of Thrones ${ }^{6}$. Dès lors en perte d'audience, les chaînes hertziennes se sont vues contraintes de lui emboîter le pas alors qu'elles ne disposaient ni des mêmes moyens ni de la même liberté. Il y a donc eu une surenchère importante autant que déséquilibrée; celle-ci invite d'une part à relativiser la morbidité supposée des spectateurs contemporains, et d'autre part, à mieux comprendre la qualité des « copies » que sont Bones et Body of Proof diffusées sur le réseau hertzien.

\section{Un discours global}

7 La dynamique du marché, dans le bouillonnement de ses offres et de leur diffusion, pourrait laisser penser que le genre et la forme des séries relèvent seulement d'une opportunité économique à saisir avant qu'elle ne disparaisse, écartant alors toute levée de sens dans un paysage sériel chaotique et informe. Pourtant, le désordre apparent n'obscurcit pas tout à fait les linéaments d'un discours global qui reflète les nouvelles obsessions et les nouveaux fantasmes des sociétés occidentales en voie de désindustrialisation. Il n'est pas indifférent de noter que les séries macabres font suite aux séries policières que tout le monde s'accorde à qualifier de séries réalistes en tant qu'elles reflètent visuellement la désagrégation sociétale : il s'agit par exemple de Law and Order ${ }^{7}$, NYPD Blue ${ }^{8}$, The Wire ${ }^{9}$ ou encore The Shield ${ }^{10}$. Dire deux mots de chacune d'elles permettra de mieux comprendre la qualité différentielle de Dexter, Bones et Body of Proof. Si, de l'avis de Marjolaine Boutet, la série politico-judiciaire Law and Order s'avère être " une véritable caisse de résonance des débats légaux et éthiques qui agitent la société américaine et un véritable document sur l'évolution de la ville de New York» (2010: $\S 45$ ), son réalisme relève du logos plus que de l'image. Pour cela, il est préférable de se tourner vers les grandes reconstitutions de terrain qui déplacent dans des univers fictifs des quartiers et des tranches de réalité consommables visuellement. NYPD Blue, par exemple, décrit le quotidien d'un commissariat de police de la $15^{\mathrm{e}}$ circonscription de New York en s'attachant de façon appuyée à la psychologie des inspecteurs, tout particulièrement l'un d'eux, Andy Cipowicz, flic irascible, alcoolique et raciste dont NYPD Blue raconte également la rédemption. The Shield s'inspire de la brigade antigang C.R.A.S.H., à Los Angeles. Elle officiait - sévissait - dans le quartier défavorisé du Rampart et ses exactions dès lors qu'elles furent connues, ont provoqué le scandale du même nom. Vic Mackey, le personnage principal de la série, en poste dans le quartier de Farmington, incarne l'essentiel de la politique de la brigade, plus proche des délinquants que de la loi. Mais c'est peut-être avec The Wire - dont l'action se déroule à Baltimore - que l'exigence réaliste atteint ses sommets. Son propos se distingue de celui des séries précédentes en ce sens qu'il rend compte de la criminalité à travers l'expérience de ceux qui la vivent: policiers, dealers, gangs, lycéens, enseignants, commerçants. L'inspecteur Ed Burns, au service de la police de Baltimore, enseignant dans les quartiers défavorisés de la ville, 
aujourd'hui écrivain et scénariste, en fut l'inspirateur; il a travaillé en étroite collaboration avec le créateur, David Simon, autrefois journaliste du crime au Baltimore Sun. The Wire a bénéficié également du concours de sociologues et d'anthropologues urbains pour que soit parachevée sa dimension réaliste.

8 Le souci de la réalité politique, économique et sociale est manifeste; la décomposition sociétale est attestée par la révélation d'un pourrissement profond qui affecte les derniers endroits où l'on s'attend à le trouver, c'est-à-dire les lieux où l'ordre prévaut. C'est la ville même - New York, Los Angeles, Baltimore - qui est la véritable victime, sorte de corps malade dont les organes vitaux sont atteints, futur cadavre offert aux regards et encore palpitant.

9 L'emboîtement des discours entre les séries policières réalistes et les séries policières macabres s'opère en deux endroits. En premier lieu, Dexter, Bones et Body of Proof peuvent bénéficier de la même caution réaliste car elles sont également issues de l'expérience du terrain. La série américaine Dexter est tirée du roman de Jeff Lindsay, Darkly Dreaming Dexter ([2004] 2006). L'ouvrage s'inspire de l'histoire de Manuel Pardo, un agent de la police de Miami, cocaïnomane, néonazi, et tueur de dealers. Il a fait au moins treize victimes et a lui-même demandé à son procès que la peine capitale lui soit appliquée : « je ne suis pas un criminel, je suis un soldat. En tant que soldat, je demande à recevoir la peine de mort. J'ai accompli ma mission ${ }^{11} »$. Manuel Pardo avait pour habitude de photographier ses victimes, de conserver les balles et de tout consigner dans son journal intime. Il a subi l'injection létale le mardi 11 décembre 2012. Ceux d'entre nous qui connaissent Bones ont du mal à croire que Temperance Brennan puisse être autre chose qu'une créature télévisuelle tant elle est stéréotypée. Pourtant, c'est l'anthropologue judiciaire Kathy Reichs qui l'a créée et elle se projette dans ses ouvrages comme dans la série dont elle est la productrice. Quant au Docteur Hunt, notre légiste " généraliste », elle ne peut se prévaloir d'une ascendance aussi directe que les deux autres. Toutefois, elle incarne partiellement à l'écran le personnage de Kay Scarpetta, médecin légiste, issue de l'expérience de Patricia Cornwell. D'abord journaliste des crimes et des faits divers au Charlotte Observer, Patricia Cornwell est devenue informaticienne à l'institut médico-légal de Richmond. La directrice de la morgue lui a inspiré le personnage de Scarpetta qu'elle a complété avec des pans importants de sa biographie et de sa personnalité. Toutes semblables et toutes différentes qu'elles soient, ces séries, à l'instar des précédentes, sont le reflet très précis d'une expérience et d'un univers réel à la télévision.

Mais l'articulation la plus significative entre le discours policier et le discours macabre est peut-être celle-ci : les séries macabres commencent là où s'arrêtent les séries policières. Une fois que le tissu des causes directes et profondes a été déployé pour produire le cadavre, il ne se trouve plus rien à dire au-delà, plus rien à montrer si ce n'est montrer et dire le résultat ; or, le résultat, c'est, au propre comme au figuré, la dépouille, terme qu'on préférera à celui de défunt pour mieux faire entendre la qualité particulière de déchet qui lui est attaché. Le corps arrive dans les laboratoires et les salles d'autopsie dans des états qui ne doivent que peu de chose à la décomposition naturelle. Le voici éviscéré, démembré, écorché, brûlé, tronçonné, et même liquéfié : un fameux épisode de Bones le sert dans une baignoire telle une soupe - une Ursuppe - où flottent quelques éléments solides que les experts repêchent avec force passoires, filtres et entonnoirs. Pareille atteinte, outrageante autant qu'outrancière, achève la déshumanisation du corps objectivé en matière putrescible, réalisant au plan charnel le pourrissement qui s'est accompli au plan urbain. 
11 Dans l'exacte mesure où la série macabre organise un au-delà de la mort, elle en recule également les limites; il y a une vie après la mort. Particulièrement visible dans Bones et Body of Proof, le bénéfice collatéral de la résolution de l'énigme - qui n'est pas le moindre -, c'est d'accomplir le chemin à rebours. Que le cadavre soit le point de départ d'un nouvel épisode ressortit certes des contraintes et des exigences du genre policier, dans le roman comme dans le film et la série, mais force est de reconnaître que cette vieille structure narrative conduit déjà à présenter la mort comme une origine. Les séries macabres, quant à elles, amplifient considérablement ce topos structural jusqu'alors cantonné en marge de l'enquête pour en faire un phénomène de premier plan. En premier lieu, la dépouille est réinvestie d'une fonctionnalité que son statut de défunt - hors d'usage - ne pouvait a priori préserver. Le cadavre recèle une vérité que l'autopsie va révéler; tel est bien le sens de Body of proof que l'on pourrait traduire par « le corps de la démonstration ». Il s'ensuit, entre les légistes et la victime un dialogue dont les termes excèdent ceux de la science pure car il se double d'un pacte également éthique : au-dessus de la porte qui s'ouvre sur la morgue où officie le docteur Hunt, on peut lire ces mots filmés à de multiples reprises : hic est locus ubi mortui docent vivos («ici est le lieu où les morts enseignent aux vivants »). (Euvrant sous ce frontispice qui invite au respect, la série Body of proof, dernière-née des trois œuvres à l'étude, s'engage dans une véritable reconstruction de la victime en lui restituant son identité et son apparence humaine : elle répare les morts. Des plans quasi rituels viennent s'intercaler dans le processus d'enquête, montrant le corps soigneusement lavé, patiemment recousu, puis recouvert enfin d'un drap blanc immaculé. Dans le contexte commercial et concurrentiel des séries, il s'agit là d'une réponse qui vaut comme rédemption pour les péchés commis contre le corps des œuvres précédentes.

\section{La vaccine réaliste}

On pourrait donc conclure que les séries macabres haussent d'un cran l'exigence réaliste $\mathrm{du}$ pourrissement parce qu'elles exposent le corps mort durant tout l'épisode. Mais, comparé à The Wire, leur réalisme est paradoxal. Montrant le cadavre, ces séries évacuent du même coup le récit réaliste alors même que leur origine les en créditait. La structure narrative de Dexter est à cet égard significative. L'épisode démarre par le rappel en quelques images nerveuses des épisodes précédents, puis vient le générique - une œuvre en elle-même - avant que n'arrive enfin Dexter sur les lieux du crime, donnant le coup d'envoi d'une nouvelle aventure. Ce point mérite d'être souligné : traditionnellement, les séries policières font état des conditions dans lesquelles le corps a été découvert : c'est notamment le cas des Experts (C.S.I. Las Vegas, New York ou Miami). Un décor extérieur est planté : un parc, l'arrière d'un magasin, une rue, où des protagonistes qu'on ne reverra plus se sont livrés à des activités innocentes, courir, faire des courses, sortir le chien, rentrer chez soi. Bref, une vision de la normalité tout d'un coup souillée par l'intrusion de l'innommable, de l'insupportable, de l'anormal : un meurtre. Cette rapide présentation du contexte contracte le réel en une citation où l'ordre américain se trouve subtilement menacé. Dans Dexter, ce préambule familier ne figure plus; de la ville qui a produit le cadavre, en l'occurrence Miami, le spectateur saura peu de chose, le réel urbain n'affleurant plus qu'à l'arrière-plan comme lien ténu de causalité. Body of proof se fait plus radicale encore et ne montre de Philadelphie que des vues aériennes; quant à Bones, on finit par oublier qu'il s'agit de Washington tant le nom de la ville est peu prononcé. La 
partie ne se joue donc plus sur ce terrain de la réalité urbaine qui ne subsiste qu'à l'état d'affiche.

Le statut du couple victime/assassin participe également à cette éviction, surtout lorsqu'il s'agit d'un tueur en série. Tous deux demeurent anonymes aussi longtemps que l'exige le suspens de l'épisode; leur histoire personnelle, dès lors qu'elle est connue, ne sera pas développée plus avant. Ils vivent donc dans une forme d'inexistence, l'un par rapport à l'autre, étroitement interdépendants. Tant que le tueur en série n'est pas identifié et que l'on n'a pas mis au jour ses motivations, il endosse le rôle de la mort personnifiée, insaisissable, non localisable, mais présente au milieu des vivants, tandis que le cadavre singulier incarne toutes les victimes potentielles. De ce point de vue, les séries - et tout particulièrement Dexter - semblent transposer à l'écran l'imagerie médiévale de la danse macabre dont elles partagent quelques traits. Dexter n'offre naturellement aucune représentation de farandole, pourtant la mort en fait courir tous les protagonistes d'un bout à l'autre de la ville. C'est elle qui mène la danse en semant les énigmes d'un vaste jeu de piste dont elle garde la maîtrise; dans Le Tueur de glace, par exemple, la tête d'une poupée Barbie est disposée dans divers lieux «froids " pour signaler l'imminence de la catastrophe et la proximité du tueur. L'obligation de décoder ce message de mort surréaliste, "[beau] comme la rencontre fortuite sur une table de dissection d'une machine à coudre et d'un parapluie!» (Lautréamont [1869] 1938: 25), se substitue au dialogue entre les mourants et la mort tel qu'il figure dans les danses macabres : il s'agit ici de traverser les signes. Notons que la supplique du mourant adressée à la mort figure littéralement dans la série lorsque Dexter s'apprête à accomplir son œuvre et que le condamné demande grâce. Bones, quant à elle, s'approche parfois très près du genre de la danse macabre quand elle place ses squelettes dans des postures grotesques. On pourrait donc accréditer la thèse selon laquelle la mort ayant disparu de nos sociétés elle ferait retour, selon nos vœux, à la télévision.

Mais, précisément, le tueur est découvert. C'est un homme - ou une femme - d'une intelligence supérieure, chargé d'une histoire hors norme et les crimes commis finissent par être justifiés par une pathologie psychotique ou un traumatisme ancien. Dexter a pris le goût du sang pour avoir été enfermé auprès de sa mère assassinée, dans un container baigné du sang maternel: le dernier arc narratif repose sur la lente remontée de ce souvenir occulté. La causalité fait donc office de raison et la raison déjoue l'arbitraire de la mort jusque dans l'absurde. Les modalités selon lesquelles le crime est commis ressortissent de protocoles extraordinairement sophistiqués d'où le hasard est banni ; les victimes de Dexter sont des criminels, celles du Tueur de glace sont des "putes", quant aux autres, celles de Bones et de Body of proof, elles doivent tout de même leur sort à un pan ou à un détail de leur biographie : une profession, une couleur de cheveux, etc. La mort ne frappe pas aveuglément, ses cibles sont choisies, et dans une certaine mesure, celles-ci sont responsables d'avoir attiré son attention. À cet égard, la façon dont on meurt dans les séries macabres rejoint bien d'autres discours de société sur la santé : le sida, l'alimentation, le tabac, pour ne citer que les plus martelés. La leçon de vanité que l'on pourrait concéder aux séries macabres s'en trouve, à la toute fin de l'épisode, retournée comme un gant : à mort d'exception, victime d'exception. Le spectateur, lui, en réchappera. Telle est la « vaccine » des séries macabres. 


\section{Leçons d'esthétique}

15 Que reste-t-il du réel dès lors qu'on en a évacué la chair ? Il reste d'une part le cadavre et d'autre part, ceux qui s'en occupent à huis clos. Ainsi qu'on l'a évoqué plus haut, les experts scientifiques, légistes, anthropologues judiciaires et autres spécialistes, officient à la morgue ou en laboratoire, dans des espaces détachés de tout, du réel comme des souillures extérieures. L'asepsie des lieux, lisses et brillants, convient bien aux occupants, lisses et brillants eux-mêmes : la particularité des héros de séries macabres réside en ceci qu'ils ne sont pas tout à fait humains, du moins qu'ils ne partagent pas notre commune humanité. Ils sont en effet tous affectés - peu ou prou - de la même étrange maladie, à savoir la forme la plus romanesque du désormais fameux syndrome d'Asperger. Promu comme un bien désirable dans la trilogie de Stieg Larson, Millenium, et avant cela, par le personnage de Lucy chez Patricia Cornwell, l'Asperger est devenu un topos de la série macabre. Tempérance Brennan (Bones) est une Asperger diagnostiquée de même que Zack, un de ses collaborateurs. Outre leur mémoire quasi eidétique et leur prodigieuse intelligence, les Asperger des séries sont dotés d'une «absence d'empathie » haussée au rang de vertu supérieure : celle-ci leur permet d'exploiter sans entrave leurs capacités mais elle les amène également à réapprendre à vivre parmi les hommes; on serait ici tenté de dire "parmi les vivants», formule qui en ferait ipso facto des morts. Ce statut intermédiaire vaut plus comme écran qui montre en empêchant de voir et moins comme intercession véritable : médiatisée deux fois, la mort est accommodée.

Le terme "réalisme » a été employé jusque-là pour désigner une catégorie de la réalité bien précise : urbaine et souillée. Rappelons, avec Ian Watt, que :

[le terme de] « Réalisme » fut apparemment employé pour la première fois comme désignation esthétique en 1835 pour indiquer « la vérité humaine » de Rembrandt en opposition à «l'idéalité poétique » de la peinture néoclassique; il fut consacré plus tard comme terme spécifiquement littéraire par la création en 1856 de Réalisme , journal édité par Duranty. (Watt $1982: 13$ )

Or, la référence à Rembrandt nous intéresse d'autant plus qu'il a peint en 1632 La Leçon d'anatomie du docteur Tulp. La toile montre un groupe de sept médecins en train d'observer la dissection que le docteur Tulp accomplit sur le cadavre d'Aris Kindt. Ces opérations, pratiquées sur des condamnés à mort, se déroulaient en public dans un theatrum anatomicum, c'est-à-dire une construction en bois à gradins étagés. Dans l'œuvre de Rembrandt, l'amphithéâtre d'anatomie n'est que suggéré mais il est loisible d'imaginer que le public se tient où se tient le peintre, vraisemblablement sur le premier gradin comme l'indique la légère contre-plongée. À l'exception d'un seul, le regard des médecins n'est pas dirigé sur l'opération en cours mais s'échappe hors champ en direction des spectateurs, intégrés dans la scène sans être représentés. C'est là une forme de présence in absentia qui préfigure la distribution contemporaine de la consommation macabre télévisuelle.

Il n'est pas exagéré de dire que Dexter, Bones, et Body of proof suivent les codes de la peinture et plus particulièrement celle de Rembrandt, d'autant que sa leçon d'anatomie n'est pas aussi réaliste qu'elle semble. Rembrandt a commencé par l'avant-bras droit de son «patient » et non par l'abdomen comme il devrait le faire si l'on en croit l'usage et l'étymologie du terme « anatomie ». W.G. Sebald, dans son ouvrage Les Anneaux de Saturne, disséquant à son tour le cadavre d'Aris Kindt, souligne ce point et ajoute que les tendons 
mis à nu dans l'avant-bras gauche et la paume de la main correspondent en fait à ceux du dos de la main droite : il en conclut que la représentation de Rembrandt va à contrecourant d'une exigence réaliste au profit d'un choix esthétique, en l'occurrence « humain»:

La main difforme témoigne de la violence qui s'exerce à l'encontre d'Aris Kindt. C'est avec lui, avec la victime et non avec la guilde des chirurgiens qui lui a passé commande de ce tableau que le peintre s'identifie. Lui seul n'a pas le froid regard cartésien, lui seul perçoit le corps éteint, verdâtre, voit l'ombre dans la bouche entrouverte et sur l'œil du mort. (Sebald [1998] 1999 : 28)

Bien qu'un tel commentaire scientifique ait été battu en brèche par des spécialistes et non des moindres (Damasio 2003), le regard de Sebald sur l'œuvre de Rembrandt corrobore l'idée d'un divorce scopique au sein de la représentation macabre : ce qui est montré n'est pas ce que l'on voit. La dissection de la main, si elle prétend bien être le sujet du tableau ainsi que le veut le titre, n'est pas, au fond, la leçon. En témoigne la façon dont la lumière est distribuée sur la toile: le corps est éclairé sur son flanc droit jusqu'à en devenir transparent et radieux - laissant penser que c'est de lui qu'émane la lumière - cependant que l'avant-bras et la main gauches demeurent dans une ombre relative.

On observe dans les séries contemporaines un même dérapage entre le projet et sa mise en œuvre. Argument scientifique de la série, l'autopsie n'est d'aucune utilité véritable dans l'économie de l'intrigue (on pourrait tout aussi bien ne pas voir de cadavres : dans les policiers de jadis, quelqu'un arrivait du « labo » et donnait les résultats). Reste donc le reste: la monstration du cadavre. Tout comme dans la peinture de Rembrandt, cela suppose un cadre, des angles, un parti pris, aussi bien politique qu'esthétique. En ce qui concerne la dimension politique, le ton est donné d'emblée par la FCC qui interdit que soit montrée la nudité. Aussi, ne verrons-nous jamais le corps entièrement dénudé, soit qu'il est dérobé aux regards par l'artifice de la prise de vue, soit qu'il est partiellement recouvert d'un drap : semblablement, dans l'œuvre de Rembrandt, un linge blanc occulte les parties génitales. Concernant la dimension esthétique, les plans "respectent" l'organisation de la peinture : le téléspectateur occupe devant son écran la position qui était celle du public de l'amphithéâtre anatomique; celle des experts scientifiques correspond, quant à elle, à celle des médecins groupés à la tête de la table d'autopsie. Le docteur Hunt, officiant principal de Body of proof, se tient généralement au chevet du défunt, à la place du docteur Tulp, et comme lui, elle indique à ses collaborateurs ce qu'il convient de voir. Toutefois, elle ne pratique jamais visiblement l'incision en $\mathrm{Y}$; cette dernière est suggérée par l'amorce d'un geste qui restera suspendu, mais qui sera complété par deux autres, également suspendus : on la voit d'une part rabattre le derme sur le torse et d'autre part recoudre en gros plan l'incision, de manière à ce que ne soient visibles que les lèvres de la plaie, la grosse aiguille et le fil. Répétés d'épisode en épisode, figés tant les plans sont brefs, ces gestes font « tableau ». figurent ses outils de travail, à savoir les photographies où se déclinent, rouges sur fond blanc, tous les types de projection de sang, encadrés comme aux cimaises d'une galerie d'art : éclaboussures, jets puissants, auréoles, etc. « On dirait du Jackson Pollock », tel est le propos, entendu dans une œuvre franco-américaine récente, Taxi Brooklyn, qui pourrait s'appliquer à cette galerie d'un nouveau genre si la couleur des œuvres n'eût été exclusivement écarlate. Dans la même gamme chromatique, citons également les installations spatiales obtenues lorsqu'il s'agit pour Dexter de visualiser les trajectoires d'objets ayant provoqué une hémorragie " pittoresque ». Le premier épisode du Tueur de 
glace est centré sur le meurtre d'un couple ${ }^{12}$. Sur la scène du crime - un appartement ultramoderne, entièrement blanc, y compris le mobilier immaculé - Dexter a installé un jeu complexe de fils rouges tendus à partir des projections de sang afin de déterminer l'endroit d'où les coups ont été portés. Ces « installations » récurrentes sont en ellesmêmes des œuvres dont la qualité esthétique est redoublée par leurs photographies curieusement présentées sur chevalet. S'exprime là un rapport volontiers distendu entre le corps absent et les traces énigmatiques qu'il a laissées malgré lui. Les deux premiers épisodes de la saison intitulée Le Tueur de glace donnent la mesure du projet. La première victime comme les autres qui vont suivre n'est plus à proprement parler un cadavre lorsqu'elle est découverte. Elle a d'une part été vidée de son sang - ce qui, pour Dexter, est un motif de chômage technique : pas de sang, pas d'expertise - et d'autre part, elle a été tronçonnée en morceaux, lesquels, emballés et ficelés dans du papier kraft, lui confèrent l'allure d'un mannequin de dessin articulé. Il y a donc là un travail plastique sur le corps, qui le dénature et interdit toute compassion. Les commentaires élogieux qui accompagnent la découverte attestent non seulement que le meurtre est un travail, mais qu'il résulte d'une recherche plastique approfondie et conceptuelle : «Pas de sang; quelle merveilleuse idée, [...] je n'avais encore jamais vu de cadavre aussi asséché, propre, net, une splendeur ${ }^{13}$.»

L'expertise scientifique de Dexter renvoie à bien des courants d'expression plastique : il ne s'agit plus de cadavre montré de façon esthétisante, mais plus radicalement d'un détournement esthétique du cadavre. Il est loisible de songer à la fois aux références plastiques du Body art et aux travaux de plastination de Gunther van Hagens. Toutefois, la surenchère dans la compétition macabre ne signifie pas que le discours sur la mort a évolué et qu'un quelconque pas a été franchi à cet égard. Qu'il s'agisse des tableaux anatomiques de Body of proof, des squelettes « rigolards » de Bones et des performances de Dexter, les séries prolongent les représentations anciennes de la mort dans des directions qui ont déjà été explorées. Le dialogue entre les vivants et les morts est figé en ceci qu'il s'obstine à montrer de quelque façon que ce soit « l'image de ce que l'on n'est pas » (Des Aulniers 2009: 171) dérobant aux regards l'image de ce que l'on sera. La chapelle napolitaine de Sansevero en matérialise l'éternelle immobilité. Dans la première salle, est exposé un Christ de marbre voilé cependant que dans la suivante se dressent deux mystérieuses «machines anatomiques ", non pas deux corps, mais l'écheveau embrouillé de leur système veineux accroché au squelette. Réalisées par le docteur Giuseppe Salerno pour Raimondo de Sangro, prince de Sansevero, ces créatures n'ont pas livré leur secret ${ }^{14}$. Entre le Christ voilé d'un marbre tellement travaillé qu'il en devient transparent et l'expression de corps réduits à la circulation figée du sang, s'étend toute l'ombre portée sur la représentation de la mort. 


\section{BIBLIOGRAPHIE}

Boutet, Marjolaine, 2010, « Soixante ans d'histoire des séries télévisées américaines », Revue de recherche en civilisation américaine, [En ligne], http://rrca.revues.org/248, mis en ligne le 29 juin 2010, consulté le 23 mai 2013.

Colonna, Vincent, 2010, L’Art des séries télé, Paris, Payot, coll. « Documents ».

Damasio, Antonio R., 2003, Spinoza avait raison : joie et tristesse, le cerveau des émotions, Paris, Odile Jacob.

Des Aulniers, Luce, 2009, La Fascination : nouveau désir d'éternité, Québec, Presses de l'Université du Québec.

Dexter, The Ice Truck Killer (Le Tueur de glace), épisode 1, saison 1, 2006.

Lautréamont, Comte de, [1869] 1938, Les Chants de Maldoror, chant 6, strophe 1, Euvres complètes, Paris, Guy Lévis Mano.

Lindsay, Jeff, [2004] 2006, Ce cher Dexter, trad. Sylvie Lucas, Paris, Points.

Mercklé, Pierre et Dollé, Thomas, 2009, « Morts en séries : représentations et usages des cadavres dans la fiction télévisée contemporaine », Raison Publique, nº 11, p. 229-246.

Ovalle, David, 2012, « Manuel Pardo: The saga of a Sweetwater ex-cop convicted of mass murder, now set for execution », Miami Herald, 8 décembre 2012, [En ligne], http://

www.miamiherald.com/2012/12/08/3133320/the-saga-of-sweetwater-ex-cop.html.

Sebald, W. G., [1995] 1999, Les Anneaux de Saturne, trad. Bernard Kreiss, Arles, Actes Sud.

Soldini, Fabienne, 2010, La mort charnelle et le cadavre dans la production artistique contemporaine : représentations, utilisations et limites du corps humain, [Research Report] CNRS Lames, [En ligne], http://www.upmf-grenoble.fr/fabienne-soldini-172205.htm, consultée le 2 juin 2013.

Watt, Ian, 1982, «Réalisme et forme romanesque », dans G. Genette et T. Todorov (dir.), Littérature et réalité, Paris, Seuil, coll. « Points », p. 11-46.

\section{Bibliographie complémentaire}

Allen, Michael, 2007, « This much I Know », dans M. Allen (dir.), Reading CSI. Crime TV under the microscope, Londres, New York, IB Tauris, p. 3-14.

Esquenazi, Jean-Pierre, 2009, La Vérité de la fiction. Comment peut-on croire que les récits de fiction nous parlent sérieusement de la réalité ?, Paris, Hermès Lavoisier.

-, 2009, Mythologie des séries, Paris, Cavalier bleu.

-, 2010, Les Séries télévisées : l'avenir du cinéma ?, Paris, Armand Colin.

Jost, François, 2001, « Séries policières et stratégies de programmation », Réseaux, n 5, p. 148-170.

-, 2011, De quoi les séries américaines sont-elles le symptôme?, Paris, CNRS Éditions. 
Turnbull, Sue, 2007, «The hook and the look. CSI and the aesthetics of the television crime series », dans M. Allen (dir.), Reading CSI. Crime TV under the microscope, Londres, New York, IB

Tauris, p. 15-32.

\section{NOTES}

1. Nous nous en tenons ici aux séries d'origine américaine, diffusées sur les chaînes du réseau français aux heures de grande écoute.

2. La série américaine Dexter a été créée en 2006 par Timothy Schlattmann; elle est diffusée aux États-Unis par la chaîne câblée Showtime. Sa première diffusion française sur Canal + date de 2007 ; elle est ensuite programmée sur TF1 en 2010.

3. À propos de l'architecture de Dexter, et particulièrement des premiers épisodes, voir Vincent Colonna (2010).

4. Bones a été créée en 2005 ; elle est actuellement diffusée aux États-Unis sur la chaîne Fox du network (réseau hertzien). Sa première diffusion française sur M6 date de 2007. On peut encore la voir sur cette chaîne en 2014.

5. Body of proof a été créée en 2010 par Christopher Murphey et Matthew Gross ; elle a été diffusée pour la première fois en 2011 sur la chaîne hertzienne ABC. Canal + a assuré sa diffusion française en 2011, puis M6 l'a programmée à partir de 2013.

6. HBO ou Home Box Office, Time Warner, 1972.

7. Law and Order est diffusée sur la chaîne NBC du réseau hertzien. Elle est diffusée en France sous les titres de New York, police judiciaire et New York District.

8. NYPD Blue est diffusée sur la chaîne câblée ABC. Elle a été diffusée en France sous le titre NYPD Blues.

9. The Shield a été diffusée sur la chaîne hertzienne FX (Fox Extended Network). Elle a été diffusée en France sous ce titre.

10. The Wire a été diffusée sur la chaîne câblée HBO. Elle a été diffusée en France sous ce titre.

11. "I'm not a criminal. I'm a soldier. As a soldier, I ask to be given the death penalty. I accomplished my mission" (Miami Herald, 8 décembre 2012, [En ligne], http:// www.miamiherald.com/2012/12/08/3133320/the-saga-of-sweetwater-ex-cop.html).

12. Dexter, The Ice Truck Killer (Le Tueur de glace), épisode 1, saison 1, 2006.

13. Dexter, The Ice Truck Killer, Le Tueur de glace, saison 1. Ajoutons que Dexter à propos d'un meurtre de «premier niveau» aura ces mots: «[c'est] peint avec les doigts, plein de dégoulinures ».

14. La réalisation de ces «œuvres » est estimée autour de 1763. Il est possible que les «sujets » aient subi de leur vivant l'injection d'une solution qui aurait coagulé leur sang.

\section{RÉSUMÉS}

La mort a disparu des sociétés occidentales en ce sens qu'elle y est devenue invisible. Est-ce la raison pour laquelle elle fait retour à la télévision dans les séries fondées sur l'autopsie? La monstration du cadavre qui ne participe en rien à l'économie de l'intrigue invite à conclure en ce sens. En corollaire, devant ces images souvent insoutenables, il serait tentant de conclure 
également à un retour d'un réalisme au-delà duquel rien d'autre ne peut être dit. Toutefois, le tableau supposé réaliste est réinvesti dans le champ esthétique, dénaturant ainsi la mort qu'il devait montrer.

Death has disappeared from Western societies in the sense that it has become invisible. Is this the reason why it reappears now in autopsy-based television series? The exhibition of the corpse which does not participate in any way in the economy of the plot invites to conclude accordingly. Considering these often unbearable images, it would also be tempting to conclude to a return to a realism beyond which nothing else can be said. However, the scene which is assumed to be realistic is reinvested in the aesthetic field, transforming the death it was supposed to show.

\section{INDEX}

Mots-clés : séries télévisées, réalisme, mort, esthétique, Dexter, Bones, Body of Proof Keywords : television series, realism, death, aesthetics

\section{AUTEUR}

JACQUELINE GUITTARD

Université de Picardie Jules Verne 